\title{
Un théâtre d'expression féminine en Ontario français depuis 2008 : étude de trois auteures
}

\author{
François Paré \\ University of Waterloo
}

Depuis 2008, les éditions Prise de Parole ont fait paraître, dans leur collection consacrée au théâtre, pas moins de vingt-quatre textes dramatiques, dont plusieurs ont pour auteurs des dramaturges appartenant à la relève littéraire franco-ontarienne. ${ }^{1}$ Cette production soutenue

\footnotetext{
${ }^{1}$ Ayant pignon sur rue à Sudbury (Ontario), les éditions Prise de parole se sont donné pour mandat « de stimuler, à partir de sa situation en région, le travail de création littéraire en milieu minoritaire et de réflexion portant sur ces milieux » (URL : https://www.prisedeparole.ca/a-propos/. Outre les textes de nouveaux dramaturges, l'importante collection « Théâtre » comprend également des œuvres plus établies, comme celles de Michel Ouellette et d'Herménégilde Chiasson, de même que des traductions. Avec Dramaturgies éditeur,
} 
FRANÇOIS PARÉ, «Un théâtre d'expression féminine en Ontario français depuis 2008 : étude de trois auteures »

témoigne, il va sans dire, d'un théâtre dont le dynamisme, notamment à Ottawa, n'a pas fléchi

au cours des dernières années, en dépit des fluctuations socio-économiques auxquelles font

face les artistes de la scène dans la plupart des sociétés actuelles. ${ }^{2}$ Ces publications relèvent

aussi d'une volonté de consigner les diverses productions théâtrales dans des versions

textuelles définitives qui en assurent la transmission et la pérennité littéraire. Si Lucie Hotte

et François Ouellet notent le défaut de relève littéraire à l'heure actuelle en Ontario français,

(2016, p. 7) ce constat ne peut donc s'appliquer au théâtre qui constitue à ce jour un des axes

les plus robustes de la littérature franco-ontarienne récente. ${ }^{3}$

\section{Une dramaturgie franco-ontarienne au féminin}

Ce renouvellement est d'autant plus intéressant que la grande majorité des dramaturges de la jeune génération franco-ontarienne sont des femmes, Écrivaines, elles sont toutes également, d'Anne-Marie White à Lisa L'Heureux ${ }^{4}$, praticiennes de la scène. Ce théâtre au féminin,

maison fondée en 1996 à Montréal par Louis-Dominique Lavigne, Prise de parole est l'un des plus importants éditeurs franco-canadiens d'œuvres pour la scène.

${ }^{2}$ En 2013, le comédien québécois Benoît Brière sonnait l'alarme. « Le théâtre est en danger », disait-il : un prognostique que reprenait à son compte le Conseil québécois du théâtre. Voir François Lévesque, «Cri du cœur du Conseil québécois du théâtre », Le Devoir, 5 avril 2013, en ligne :

https://www.ledevoir.com/culture/theatre/374982/cri-du-coeur-du-conseil-quebecois-du-theatre.

Bien que les recherches de Dominique Pasquier ne rendent compte que du milieu théâtral français, les conclusions de cette chercheure sur la transformation des sociabilités et des habitudes de fréquentation du public de théâtre s'appliquent sans aucun doute au contexte canadien. Voir : Dominique Pasquier, «Sociabilités et sorties au théâtre », Culture études, vol. 1, no 1, 2013, p. 1-12, disponible en ligne : https://www.cairn.info/revue-culture-etudes-2013-1-page-1.htm, page consultée le 25 janvier 2019.

${ }^{3}$ Outre ces remarques préliminaires, il faut souligner que certains dramaturges franco-ontariens actuels, établis dans la région d'Ottawa-Gatineau, ne sont pas originaires de l'Ontario et ne répondent pas aux conventions habituelles dans l'établissement des corpus d'œuvres franco-ontariennes. La fluidité de la frontière culturelle et littéraire entre les deux rives de la rivière des Outaouais accentue vraisemblablement ce problème. Néanmoins, il ne fait pas de doute que les œuvres auxquelles notre étude renvoie ont été conçues et produites à Ottawa et appartiennent à l'histoire du théâtre franco-ontarien, peu importe le lieu de naissance des auteures. Publiées par un éditeur franco-ontarien, ces œuvres contribuent sans l'ombre d'un doute aux activités du Théâtre La Catapulte, du Théâtre du Trillium et du département de théâtre de l'Université d'Ottawa. Elles témoignent du pouvoir d'attraction de la relève théâtrale en Ontario français au cours de la période étudiée.

${ }^{4}$ Certaines de ces auteures dramatiques ne sont pas natives de l'Ontario. Anne-Marie White est originaire de Petit-Rocher au Nouveau-Brunswick, alors que Lisa L'Heureux est née dans l'Outaouais québécois. Elles sont toutefois actives depuis plusieurs années au sein du milieu théâtral franco-ontarien et publient leurs textes en 
rompant avec une dramaturgie largement dominée par leurs prédécesseurs masculins (André Paiement, Jean Marc Dalpé, Robert Bellefeuille, Robert Marinier, Michel Ouellette), ${ }^{5}$ évolue depuis une quinzaine d'années dans l'orbite de compagnies théâtrales d'Ottawa, dont le Théâtre la Catapulte, le Théâtre du Trillium et le Théâtre Rouge Écarlate. Les femmes jouent, en effet, depuis le début du XXI ${ }^{\mathrm{e}}$ siècle, un rôle absolument déterminant dans la création et la production de textes dramatiques originaux dans la région de la capitale fédérale. Lucie Robert note que la création sur scène d'œuvres porteuses de revendications féministes remonte au début des années 1970, au moment où émergent à Montréal certains collectifs militants, tels le Théâtre expérimental des femmes et le Théâtre des cuisines. (2005, p. 44) Or, à la même époque, les femmes ne sont certes pas absentes de la scène théâtrale émergente en Ontario, ${ }^{6}$ comme en atteste l'arrivée de Brigitte Haentjens au Théâtre du Nouvel-Ontario en 1980, mais leur contribution reste à ce jour mal représentée et assez peu étudiée.

Il est clair que cette cohorte d'auteures et de praticiennes du théâtre, toutes très actives dans les milieux culturels d'Ottawa, entretient des liens étroits d'abord avec le Théâtre la Catapulte, dirigé successivement pour la période étudiée par Louis Patrick Leroux, Joël Beddows et Jean Stéphane Roy, puis avec le Théâtre du Trillium, dont la direction est assurée tour à tour par Sylvie Dufour et Anne-Marie White, et enfin avec les départements de français

Ontario. C'est sur cette base qu'elles trouvent place dans cette étude. Par ailleurs, Sarah Migneron est originaire de Mississauga en banlieue de Toronto. Elle a fait ses études à l'école secondaire Sainte-Famille de cette ville.

${ }^{5}$ Dans sa superbe étude sur l'évolution historique du théâtre franco-ontarien, Jane Moss note d'ailleurs cette faible présence des femmes dans le théâtre franco-ontarien. Voir Jane Moss, « Le théâtre francophone en Ontario », dans Lucie Hotte et Johanne Melançon (dir.), Introduction à la littérature franco-ontarienne, Sudbury, Prise de parole, 2010, p. 83-84.

${ }^{6}$ Paulette Gagnon et, plus tard, Marie-Thé Morin jouent chacune un rôle déterminant dans la production et la promotion d'un théâtre franco-ontarien original. Gagnon œuvre à divers titres au Théâtre du Nouvel-Ontario à Sudbury de 1982 à 1996. Elle prendra ensuite la direction de La Nouvelle Scène à Ottawa (1997-2001). Comédienne, metteure en scène et écrivaine, Marie-Thé Morin est active à la compagnie Vox Théâtre (Ottawa) dès 1979. Elle est l'auteure d'une dizaine de créations pour la scène. 
FRANÇOIS PARÉ, «Un théâtre d'expression féminine en Ontario français depuis 2008 : étude de trois auteures »

et de théâtre de l'Université d'Ottawa. ${ }^{7}$ Ayant travaillé de près avec Louis Patrick Leroux au début des années 1990, White collabore ensuite avec Joël Beddows à la Catapulte. ( V. Thibault, 2008, p. 12-13) L'Heureux, Migneron et d'autres dramaturges et metteures en scène, comme Mishka Lavigne, Marie-Pierre Proulx et Caroline Yergeau, se rencontrent régulièrement dans le cadre des productions du collectif ${ }^{8}$ Les Poids Plumes, établi à Gatineau et fondé par Lisa L'Heureux ${ }^{9}$. Il s'agit donc d'un remarquable réseau de créateurs, d'acteurs et de producteurs de part et d'autre de la frontière interprovinciale.

Du côté ontarien de la rivière des Outaouais, il est clair qu'en tant que professeur à l'Université d'Ottawa et directeur du Théâtre la Catapulte, Joël Beddows joue un rôle clé dans l'émergence d'un théâtre franco-ontarien de création, écrit, produit et mis en scène par des femmes. Dans un article substantiel qu'elle lui consacrait en 2012, Stéphanie Nutting, sans faire remarquer cet impact particulier du travail de Beddows, note l'étonnante complexité de ses productions et de ses interventions auprès d'une foule d'institutions culturelles aussi bien francophones qu'anglophones en Ontario. (2012, p. 17) Inspiré à son arrivée à Ottawa par une volonté d'ouvrir le théâtre franco-ontarien aux débats en cours dans les sociétés occidentales, Beddows s'est trouvé à favoriser directement et indirectement la venue à la scène de voix nouvelles, surtout féminines.

En tenant compte de ce contexte d'effervescence des arts de la scène à Ottawa depuis une quinzaine d'années, notre objectif, dans les pages qui suivent, sera double. Il s'agira,

\footnotetext{
${ }^{7}$ Sarah Migneron, Lisa L'Heureux et Anne-Marie White sont toutes trois diplômées de l'Université d'Ottawa.

${ }^{8}$ Cette liste est loin d'être exhaustive. Il faudrait y ajouter, par exemple, les noms de leurs collègues masculins Antoine Côté Legault et Louis-Philippe Roy.

${ }^{9}$ Le collectif Les Poids plumes s'est dissout en 2018 peu de temps après le départ de Marie-Pierre Proulx et Antoine Côté Legault pour Sudbury.
} 
d'une part, d'examiner l'apport de dispositifs textuels et scéniques novateurs dans le théâtre franco-ontarien d'expression féminine dans la région d'Ottawa depuis 2008, et, d'autre part, de documenter la persistance de représentations dysphoriques liées à la maison et à la famille dans certaines œuvres de la relève théâtrale. Dans notre étude de trois dramaturges de la relève féminine, Anne-Marie White, Lisa L'Heureux et Sarah Migneron, nous serons à même de constater l'émergence d'éléments stylistiques et textuels qui repoussent, sans pourtant le désavouer, l'horizon même du théâtre franco-ontarien, tel qu'il s'était dessiné depuis les premières pièces d'André Paiement au début des années 1970 jusqu'aux œuvres marquantes de Jean Marc Dalpé, Robert Marinier et Michel Ouellette.

Les œuvres de White, L’Heureux et Migneron sont dénuées de toute référence à l'Ontario français. Malgré la persistance du drame familial, élément clé de tant d'œuvres théâtrales contemporaines au Québec et au Canada français, on assiste ici plutôt à une reformulation du spectacle scénique, désormais façonné par la perte de cohérence du récit identitaire et l'aventure introspective de chacun des personnages dans sa solitude existentielle. Ce théâtre d'expression féminine ne renvoie pas non plus, de façon explicite, à l'expérience douloureuse de la minorisation linguistique et de l'exclusion socio-économique que revendiquait le théâtre franco-ontarien depuis les années 1980. La structure actantielle et la trame narrative qu'elles privilégient supposent plutôt la mise en présence d'individualités orphelines, des « je » et des « tu » irréductibles, certes victimes d'ostracisme et de violence, mais sans autre distinction que le régime pronominal lui-même auquel s'assujettit une mémoire marquée par le traumatisme. Profondément intériorisée, la marginalité se transforme alors en condition heuristique. Chez les trois dramaturges, les personnages sont appelés désormais à assumer entièrement les conditions difficiles de leur venue à la parole. 
FRANÇOIS PARÉ, «Un théâtre d'expression féminine en Ontario français depuis 2008 : étude de trois auteures »

Dans les limites de cette étude, nous avons choisi de nous pencher tour à tour sur trois œuvres qui nous semblent représenter certaines tendances dramaturgiques marquantes en Ontario français depuis 2008. Déluge d'Anne-Marie White attirera d'abord notre attention, car cette pièce de facture assez traditionnelle nous permettra de saisir les enjeux formels et identitaires des œuvres à l'étude. En septembre 2012, le Théâtre du Trillium met en scène ce drame familial, sous la direction de l'écrivaine elle-même, Le texte de cette production charnière de la relève théâtrale à Ottawa paraîtra l'année suivante aux éditions Prise de parole. Déluge soulève la question de la stigmatisation qui hante le personnage depuis sa naissance, comme une tare débilitante. La marginalisation de l'enfant, injuste et brutale, prend la forme, chez White, d'un bannissement quasi ontologique dont les échos forment le tissu même du texte. Cette première incursion nous amènera à cerner les transformations stylistiques mises en œuvre par la dramaturgie franco-ontarienne récente, tout en remarquant la reprise de schèmes identitaires liés à la filiation. Pour l'hiver de Lisa L'Heureux, œuvre que nous commenterons ensuite, interroge les protocoles narratifs et dialogiques qui permettent d'amarrer l'expression autoréflexive de la mémoire aux formes postmodernes de la choralité. Inspirée, aux dires de la dramaturge, par la vie du poète Arthur Rimbaud, (Goffi, 2017) cette rhapsodie à trois voix suit de très près la « cohérence misérabiliste » (Nolette, 2015, p. 132) du psychodrame familial qui avait fait la fortune du théâtre franco-ontarien entre 1970 et le tournant du siècle. Enfin, une œuvre extrêmement différente sur le plan formel attirera notre attention. La pièce en vingt-quatre tableaux $\grave{A}$ tu et à moi de Sarah Migneron a d'abord été remarquée par le Théâtre la Catapulte en 2004, alors même que l'écrivaine et traductrice était inscrite au premier cycle à l'Université d'Ottawa. Cette œuvre précède donc en principe la période étudiée. Cependant, elle n’émerge véritablement dans le 
corpus considéré qu'au moment où elle est mise en scène à l'automne 2013 par la troupe de L'Atelier avec l'appui de la Chaire de recherche du Canada sur la francophonie canadienne de l'Université d'Ottawa. Elle est ensuite publiée chez Prise de parole en 2015. En dépit de cette percée plutôt lente, $\grave{A}$ tu et à moi frappe par sa forte dimension symbolique et l'originalité de ses propositions scéniques.

Les trois écrivaines reprennent à leur compte le projet de déconstruction des fondements socio-culturels de l'exclusion et de la marginalisation. Pour White et L'Heureux, le théâtre lève le voile sur les interdits et les omissions qui façonnent et entravent le sujet féminin. Dans l'œuvre de Sarah Migneron, plus proche du surréalisme, l'espace scénique interroge le langage lui-même et ses réserves d'associations libres. À chaque fois, le théâtre traduit la volonté existentielle du sujet féminin de transformer les traumatismes du passé. C'est ainsi que, comme nous le verrons à l'instant dans Déluge d'Anne-Marie White, le personnage de la femme «abîmée » et révoltée, occupant le centre de la scène, deviendra le véhicule de puissantes revendications identitaires.

\section{Déluge d'Anne-Marie White : enfantement et violence originaire}

Déluge compte huit tableaux, portant tous des intitulés dans la version publiée. ${ }^{10}$ Ces titres mettent en valeur le récit à la première personne de Solange, une « femme amochée » qui cherche dans sa mémoire les fragments de son enfance brisée. Le personnage féminin mis en scène par Anne-Marie White se définit par une profonde haine de soi et une sourde anxiété face à la maternité. Incapable de s'accepter, Solange entreprend une quête initiatique

\footnotetext{
${ }^{10}$ Anne-Marie White, Déluge, Sudbury, Prise de parole, 2013. Les références subséquentes à cette œuvre seront indiquées par la lettre $D$, suivie de la page, entre parenthèses. Ces intitulés n'appartiennent pas qu'à la version écrite de cette œuvre. Lors de sa représentation à la Nouvelle Scène en 2012, les metteurs en scène avaient opté de les faire entendre au public sous forme d'enregistrements.
} 
FRANÇOIS PARÉ, «Un théâtre d'expression féminine en Ontario français depuis 2008 : étude de trois auteures »

imaginaire, « un voyage intérieur dans un cauchemar existentiel » (Deglise, 2013) dont les étapes forment la structure de la pièce. Alors qu'elle erre dans les rues de la ville, la femme fait la rencontre d'autres êtres qui avivent chez elle un profond sentiment d'inadéquation. Se voyant confier par une voisine la garde temporaire de son enfant, l'errante se trouve tout à coup entraînée dans l'engrenage de ses souvenirs. Le rideau s'ouvre du reste sur une première scène monologique, intitulée «Je suis un déchet inodore ", au cours de laquelle Solange s'adresse à un « tu » multiple et intériorisé qui suscite les images troubles d'une «maison familiale $[\ldots]$ remplie d'absence » $(D, 8)$. L'enfant dont la voisine lui a confié la garde, le temps d'aller voir sa mère à l'urgence, ramène Solange au traumatisme de sa naissance et au refus quasi congénital de l'enfantement. Doutant de la réalité des êtres qui l'entourent, elle est alors assaillie par une suite de scènes étranges, peuplées de personnages hétéroclites qui ramèneront à la surface les fragments de son passé tourmenté. L'angoisse que Solange ressent à l'idée d'être mère évoque donc la violence larvée de sa propre venue au monde, son enfantement proprement dit. N'est-ce pas le refus de la maternité qui fait obstacle non seulement à la pleine identité, mais aussi à la perpétuation du récit inaugural sur lequel se fonde l'individualité?

L'œuvre d'Anne-Marie White se construit donc comme un ensemble de scènes spectrales et d'ellipses qui permettent au personnage central de reconstituer, par à-coups, le terrible sentiment de perte qui l'accable. Au tableau 5, lors de sa rencontre avec une passante à la recherche du «Centre de la tendre enfance » qu'elle n'arrive pas à trouver dans le dédale des rues, Solange, bouleversée par la vue de cette femme et de plus en plus isolée, oscille entre la violence émancipatrice et la norme qui lui est imposée depuis longtemps. Cependant, 
incapable de dépasser cet espoir larvé, elle finit par s'éclipser à nouveau de la scène fantasmée :

Je voudrais être heureuse pour ce jeune couple, pour leur avenir, pour leur bonheur qui s'annonce dans leur vie.

Je voudrais $(D, 49)$.

Comme on le voit, le monde ambiant ne fait que confirmer, chez ce personnage, les appréhensions de la femme meurtrie, dès sa venue au monde, par un passé de répression et de violence.

Le tableau 6 est à cet égard d'une importance capitale, car il établit le lien causal entre le traumatisme de l'enfantement chez Solange et l'expérience de la violence masculine qui conjugue explicitement la destinée de l'enfant et celle de la femme adulte. Ayant d'abord évoqué son héritage maternel, Solange fait alors la rencontre du voyou, symbole selon elle de la descente de la société tout entière dans la folie. Par son langage vulgaire et ses propos blessants, le voyou établit aux yeux de la femme la responsabilité de la voix normative masculine. C'est en elle que naît la grammaire de la violence, car la grossièreté du voyou voulant s'en prendre à une vieille dame dans la rue devient ironiquement l'incarnation vivante de la norme sociale. Solange en fait elle-même l'observation, en extrapolant la marginalité du jeune délinquant à l'ensemble des hommes :

Le monsieur va frapper la vieille dame.

Ça risque de ne pas durer bien longtemps.

Il est efficace, le monsieur...

Tu vois?

Un autre coup.

Un dernier coup.

Dans le souffle, celui-là $(D, 57)$.

Au cours de son itinérance dans les rues inquiétantes de la ville, Solange avait croisé jusquelà certains représentants de la normativité médicale. L'hygiéniste et, plus tard, le dentiste, 
FRANÇOIS PARÉ, «Un théâtre d'expression féminine en Ontario français depuis 2008 : étude de trois auteures »

avaient évoqué pour elle l'intolérance prescriptive de la propreté et de l'hygiène. Tout contrevient alors à son affranchissement sur le plan symbolique, car la souillure que la femme errante ressent dans son corps même (elle est rongée par son « hamster intérieur » $D, 29$ ) la reconduit, qu'elle le veuille ou non, aux souffrances de sa naissance, ou plutôt de toutes les naissances :

Des gémissements comme des pleurs d'enfants...

L'enfant.

Tout est à propos de l'enfant $(D, 36)$.

C'est alors qu'elle entend donner une leçon à celui-là même qui l'accompagne, elle qui, pense-t-elle, ne croit pas « avoir l'instinct maternel » $(D, 40)$.

Au cours de la pièce, le pronom «tu » est d'ailleurs graduellement associé au fantasme du garçon abandonné, nommé Dumbo, dont Solange revoit au tableau 8 les images troublantes. Le renvoi au film d'animation produit par la société Disney en $1941^{11}$ et au personnage du jeune éléphant de cirque, exploité et marginalisé, témoigne des fondements symboliques de l'errance du personnage féminin dans Déluge. Le lien inaliénable qui unit la femme et l'enfant s'oppose à la peur de l'abandon et surtout à l'abjection, évoquées dans chacun des tableaux de la pièce. Cette présence fraternelle de l'enfant n'est jamais matérialisée sur scène. Aucun acteur ne pourra venir incarner le personnage irreprésentable et pourtant crucial sur le plan symbolique de Dumbo. Pourtant, Solange voit en l'enfant

\footnotetext{
${ }^{11}$ Ce film d'animation, basé sur un scénario de Helen Aberson et Harold Pearl, raconte l'histoire d'un éléphanteau, ridiculisé pour ses grandes oreilles. Après avoir été abandonné par sa mère, Dumbo parvient par sa capacité de voler à transcender pour un temps seulement les limites de son enfance marquée par la stigmatisation. Le personnage de Dumbo est associé par les chercheurs aux représentations déshumanisantes des Noirs dans la culture populaire américaine de l'après-guerre. Voir Russell Reising, Loose Ends : Closure and Crisis in the American Social Text, Durham, Duke University Press, 1996, p. 310 et suiv..
} 
stigmatisé une chance de rédemption, car elle aussi pourra dépasser les limites de sa condition :

Je veux survoler les édifices qui m'écrasent.

Je rêve de vents violents et de cours de grammaire $(D, 48)$.

L'évocation en filigrane du film de Disney éclaire donc, dans Déluge, l'histoire tourmentée de Solange, orpheline de sa naissance proprement dite et de toute naissance.

Le palimpseste soulève également la question de la composition elliptique de cette œuvre. Le recours aux formes brèves du tableau ne signale pas, chez Anne-Marie White, le maintien d'une structure monologique que certains chercheurs, comme Lucie Robert, attribuent à la dramaturgie féminine des quarante dernières années :

[1]e monologue apparaît ainsi comme une esthétique centrale dans un théâtre qui se veut féministe. Apparenté au psychodrame, il est le lieu de la prise de conscience, de l'expression voire de la libération de la parole féminine. En effet, le monologue écrit par des femmes renonce à la construction d'une fiction et d'une intrigue, à la séparation entre la fiction et la réalité, entre la scène et la salle, au profit d'une logique participative. (2005, p. 43-46)

Sans écarter le monologue, Anne-Marie White ne s'y limite pas. Car Déluge est une œuvre où la pluralité des voix et leur entrecroisement importent particulièrement. S'il y a rupture dans la communication avec l'autre, la coprésence des personnages sur scène accentue la poly-vocalité de la trame narrative et ses effets d'exemplarité. La dramaturge faisait état de cette nécessité du fragment au cours d'une entrevue en marge de sa pièce \#PigeonsAffamés, présentée à Québec en 2016 : «C'est un format qui est très proche de la façon dont notre cerveau est formaté aujourd'hui, avec beaucoup de ruptures. C'est une mosaïque où il n'y a pas nécessairement de liens linéaires entre les différents segments, mais où un lien se dégage au fil du spectacle ». (Leclerc, 2016) D’une part, les diverses formes de la fragmentation, dont la poly-vocalité est un élément singulier, confirment, dans cette œuvre, la crise du 
FRANÇOIS PARÉ, «Un théâtre d'expression féminine en Ontario français depuis 2008 : étude de trois auteures »

dialogue théâtral qui définit l'ensemble des stylistiques de la scène depuis le milieu du XX siècle, comme le note Jean-Pierre Ryngaerts dans son étude du personnage thêâtral contemporain. D'autre part, contrairement à l'analyse proposée par ce chercheur, la succession de tableaux plus ou moins disjoints n'est pas symptomatique d'une perte généralisée « des liens entre la créature et son auteur, des liens entre la créature et une identité marquée, entre l'ancien pôle du caractère et les paroles prononcées, entre les paroles prononcées et la situation, l'action ou l'information ». (2008, p. 104) Au contraire, la juxtaposition non hiérarchisée des voix donne lieu à une corrélation des récits individuels, qui reste à la fois univoque et multiple. Une telle choralité, qui absorbe les personnages sans pour autant les fusionner, définit également, nous le verrons maintenant, la pièce Pour l'hiver de Lisa L'Heureux. Dans ce cas, la structure chorale de l'espace scénique permet une amplification polyphonique de la mémoire mutilée.

\section{Pour l'hiver de Lisa L'Heureux : la maison pleine de trous}

Comme dans Déluge d'Anne-Marie White, la succession rapide des tableaux, souvent très brefs, imite, dans le théâtre de Lisa L'Heureux, le fonctionnement sporadique de la mémoire. Pour l'hiver compte, en effet, vingt-trois scènes où se croisent les trois personnages de cette pièce dans laquelle affleurent un certain nombre d'intertextes importants, dont les premiers versets de la Genèse, des chansons de Tom Cochrane et de Patsy Cline, de même que le célèbre sonnet des Voyelles d'Arthur Rimbaud. Il arrive qu'à l'intérieur d'un seul tableau, le discours monologique se résume à la perspective unique de chacun des personnages, surtout dans le cas d'Arthur, seul au chevet de sa mère mourante. Cependant, ces intenses moments d'introspection font vite place aux voix superposées ou interposées qui, sous l'apparence du dialogue, rompent l'unité de la trame narrative, sans pour autant renoncer aux structures du 
récit. Contrairement au concept de polyphonie, élaboré par Hervé Guay dans son étude du théâtre québécois contemporain, les voix multiples mises en scène par L’Heureux ne produisent pas à proprement parler « de la divergence au sein du discours spectaculaire ». (2010, p. 18) Au contraire, l'entrecroisement des scènes obsessionnelles chez les trois personnages illustre une violence primordiale aussi terrible qu'unidimensionnelle. Car, dans ses représentations récurrentes dominées par la figure du père, la maison natale n'appartient à personne. Le propos dramaturgique est justement de la rendre universelle, comme si, à elle seule, le lieu de l'origine a accumulé dans la mémoire des personnages les traces de toutes les violences. Le foyer familial est dès lors la source de l'inadéquation même.

Pour le personnage de Valérie dans Pour l'hiver, le domicile parental évoque la présence menaçante du pronom «Lui », symbole de l'intolérance et de la norme. Or, sur scène, le rappel de la figure masculine oppressante semble déjà subir les effets de dislocation du récit :

Y a des trous dans les murs de leur maison parfaite Là où son poing l'a manquée

Des gouttes de sang

Sur le plancher de bois franc

Qu'elle essaie d'essuyer avant que ça sèche (L'Heureux, 2016, p. 45) ${ }^{12}$

Ces images troublantes produisent chez chacun des personnages un sentiment de culpabilité généralisée. Plus ils cherchent à comprendre le récit de leur naissance, plus cette quête les conduit à une faute primordiale dont ils portent encore les traces, comme une sorte de décalage au sein de leur identité. Enfermée dans une salle d'interrogatoire au poste de police, Valérie dit ne pas savoir les causes de son arrestation :

\footnotetext{
${ }^{12}$ Les références subséquentes à cette œuvre seront indiquées par la lettre $P$, suivie de la page, entre parenthèses.
} 
FRANÇOIS PARÉ, «Un théâtre d'expression féminine en Ontario français depuis 2008 : étude de trois auteures »

Écoutez-moi bien

Monsieur l'agent

Je peux pas vous dire exactement ce qui s'est passé

Parce que je le sais pas

Je sais pas ce qui s'est passé $(P, 19)$

Plus tard, en parallèle aux aveux d'Arthur sur son incapacité à trouver un sens à sa vie, la jeune femme s'en prend au système scolaire, car c'est par l'école que l'inadéquation à la norme façonne à jamais l'identité :

On s'en crisse-tu de leurs dates pis de leurs formules à marde C'est pas comme s'y [sic] aiment ça nous enseigner

La matière les écœure autant que nous

Pis anyway

Y nous considèrent tous comme des causes perdues d'avance Une génération de manqués $(P, 22)$

L'impatience de Valérie répond au caractère systémique de la dégradation du sujet, dès sa naissance. Si, dans l'œuvre de L'Heureux, le personnage tient tant à prendre la parole, même en l'absence de toute réponse, c'est qu'il ne peut envisager sa différence affirmée que sur le plan de la représentation. Bernard aura beau réciter les premiers versets de la Genèse, alors que le Créateur n'avait pas encore institué la nécessité de la faute originelle, il n’arrivera pas à endiguer les vagues de culpabilité, puissantes et indistinctes, qui les paralysent, lui et tous les autres.

Peut-être parce qu'il appartient à une époque plus ancienne, Arthur est porteur d'une conception plus large de l'inscription identitaire, même s'il avoue, lui aussi, son sentiment de faute au tableau 21, dans les derniers moments de la pièce. Pour l'instant, au chevet de sa mère mourante, il se prend plus d'une fois à rêver de leurs voyages, tous les deux, dans l'apparente liberté du continent tout entier : 
On avait un vieux Chevy Malibu qui traînait de peine pis de misère

La roulotte dans laquelle on vivait

Notre paradis mobile

Pis y avait la route devant nous

Une route interminable sur laquelle on était comme

Deux explorateurs à la découverte de nouveaux mondes

Ou comme des hippies

Très à la Jack Kerouac $(P, 25)$

Si caractéristique de la littérature du Canada francophone, le trope de la route et son intertexte (nommément Jack Kerouac et la chanson «Life is a Highway » de Tom Cochrane ${ }^{13}$ ), s'opposent aux inadéquations associées à la maison natale et à l'école. Comme chez Gabrielle Roy, la route souligne l'euphorie d'une filiation symbiotique entre la mère et l'enfant, alors que se déploie la cartographie des grandes migrations du « nouveau monde ». Cependant, le fantôme maternel sur son lit de mort emporte désormais avec lui toute possibilité de recommencement. Dans un passage important du dixième tableau, il revient au personnage de Valérie de dénoncer une fois pour toutes l'intolérance du père, ce pronom « Lui » qui radie toute chance de filiation et, donc, d'avenir.

En effet, si la souffrance et la solitude de Bernard, Valérie et Arthur transcendent les époques où ceux-ci évoluent, leur expérience respective de la violence et de l'abandon est le signe d'un terrible besoin de communiquer la souffrance vécue par tous et toutes. Dans Pour l'hiver, la faute inaugurale est d'autant plus ressentie par les personnages qu'elle est toujours

\footnotetext{
${ }^{13}$ Cette chanson de l'auteur-compositeur-interprète canadien Tom Cochrane date de 1991. Selon les données du texte, Arthur devait avoir cinq ou six ans à l'époque. Dans la pièce, c'est au tableau 6 qu'apparaissent les deux premières lignes du refrain : «Life is a Highway / I wanna ride it all night long ». Dans une entrevue récente, Cochrane évoque son désir de créer un texte positif qui insufflerait l'espoir aux jeunes de sa génération. Voir David Friend, «Tom Cochrane reflects on success of "Life is a Highway" », The Canadian Press et The Toronto Star, 5 janvier 2017, en ligne: https://www.thestar.com/entertainment/music/2017/01/05/tom-cochrane-reflects-on-success-of-life-is-ahighway.html, page consultee le 25 janvier 2018.
} 
FRANÇOIS PARÉ, «Un théâtre d'expression féminine en Ontario français depuis 2008 : étude de trois auteures »

indéterminée et diffuse. La scène est donc un espace expiatoire et le thêâtre toujours une ritualisation de la venue au monde du personnage dans son refus de ce «Lui » honni, où se logent l'intolérance et l'abjection.

En revanche, la déclamation par Bernard du célèbre poème de Rimbaud souligne, en parallèle, la puissance libératrice de la parole incarnée sur scène. Tandis que les formes brèves retenues par la dramaturge désamorcent l'unité d'un récit où s'abolissent les déchirements du passé, cet éclatement des formes n'entrave pas chez les personnages le besoin pressant de révéler les secrets enfouis dans la mémoire. Les violences liées aux structures oppressives de la famille, de même que certains moments de grâce, ne pénètrent l'espace scénique que sous forme de passages interstitiels. Les dérives monologiques dans lesquelles s'engagent à tour de rôle Arthur, Bernard et Valérie s'insèrent ici et là comme des surgissements heuristiques qui constituent le cœur du drame mémoriel. À ce titre, on peut penser que, comme les «mouches éclatantes » du sonnet des Voyelles, les personnages créés par L'Heureux «bombinent autour / des puanteurs cruelles» $(P, 48)$ de leur passé de violence. Toutefois, les éléments mémoriels sont aussitôt emportés par l'entrecroisement incessant des répliques et le désir de tout dire d'une seule traite. La poly-vocalité n'est donc pas ici une forme de la choralité : elle semble en effet receler en son centre une parole unitaire, urgente et nécessaire, la même pour tous, seule capable de mettre en œuvre une subjectivité enfin émancipée.

\section{À tu et à moi de Sarah Migneron : une esthétique du discontinu}

Inspirée sans doute par le théâtre français de l'absurde et certaines esthétiques postmodernes, la pièce $\grave{A}$ tu et à moi de Sarah Migneron fait également appel à des formes stylistiques fragmentées qui déstabilisent le spectateur, incapable de saisir par la seule voix des 
personnages l'orientation de la trame narrative. Cette œuvre se démarque des autres productions théâtrales franco-ontariennes récentes dans la mesure où elle est motivée également par le refus du réalisme psychologique et de toute représentation spatiotemporelle. Le théâtre de Migneron est donc, à prime abord, beaucoup plus abstrait que celui de White et de L'Heureux. D'une part, les deux personnages créés par la dramaturge et identifiés simplement par des tirets de longueur variable dans la version publiée ne comportent aucune autre marque distinctive qui permettrait de les situer dans le temps et dans l'espace référentiels. Comme chez Samuel Beckett, par exemple, les personnages protéens se résument au hasard de leurs apparitions sur scène et aux dimensions symboliques qui émanent parfois de la vacuité de leurs échanges. Le dialogue reste en effet résolument superficiel et sans valeur étiologique pouvant permettre au public de reconstituer l'histoire individuelle des deux devisantes. L'intégrité du personnage se dissout face à la fragmentation généralisée du récit dramatique, laissé souvent en suspens, comme si, au dernier moment, la fonction dialogique s'enrayait et que rien ne pouvait plus être dit.

Au-delà de cette structure fragmentaire se dessine la présence symbolique de l'ellipse elle-même, figure de l'omission, qui constitue la matière primordiale du théâtre de Migneron. C'est sur cette opacité du non-dit que se découpent les vingt-quatre tableaux ou propositions scéniques d'À tu et à moi et que s'inscrivent ses éléments symboliques les plus pressants. Selon Alvina Ruprecht, «[1]e texte de Sarah Migneron tient à la fois d'une partition pour voix, et d'un scénario de situations mises en espace par un chorégraphe (on parle de dramaturgie corporelle), où se mêlent les voix et les corps qui font penser aux chorégraphies de la célèbre Martha Graham » (2013). Bien que son analyse de la pièce de Migneron renvoie en grande partie à la mise en scène de Joël Beddows et Marie-Claude Dicaire à L'Atelier 
FRANÇOIS PARÉ, «Un théâtre d'expression féminine en Ontario français depuis 2008 : étude de trois auteures »

d’Ottawa, Ruprecht note néanmoins la nouveauté des modes de représentation du personnage chez cette auteure, les associant à une dramaturgie du mouvement corporel et du mime. Or, nous le verrons à l'instant, cette technique de l'ellipse se double d'un travail essentiel sur les capacités figuratives du langage.

Dès les premiers tableaux, les deux personnages féminins semblent dépourvus de profondeur. Ils sont de toute évidence interchangeables, ne faisant à tour de rôle que lancer le jeu au gré de leurs rencontres. Leurs silences, indiqués dans le texte par des points de suspension, démontrent leur difficulté à anticiper la suite des choses. Dans la version publiée, seul l'accord du participe passé révèle le genre des deux figures qui alternent ainsi dans la conduite des dialogues. Outre cette identité fugitive, il est plutôt difficile d'attribuer des traits de caractères particuliers à l'une ou l'autre des intervenantes. Jusqu'au tableau 7, celles-ci se renvoient d'ailleurs les questions sans tenter d'y répondre et échangent entre elles des platitudes ou des évidences. Il s'agit, au tableau 3, par exemple, d'un enchaînement de questions apparemment anodines sur l'atome :

--- J'ai une question pour toi.

- Une question?

--- Oui.

- Vas-y.

--- As-tu déjà vu un atome?

- Un quoi?

--- Un atome.

- C'est quoi, un atome? (Migneron, 2015, p. 12) ${ }^{14}$

Cette entrée en matière par une suite de questions incongrues ou banales structure l'ensemble des jeux de langage qui se déroulent sur scène.

\footnotetext{
${ }^{14}$ Les références subséquentes à cette œuvre seront indiquées par la lettre $A$, suivie de la page, entre parenthèses.
} 
Cependant, le texte de Migneron ne cesse de désigner ce qui, au-delà du vide, constitue la véritable matière de la pensée. Le tableau 3 donne lieu, dès lors, à une autre lecture. N'est-ce pas de cette manière que se définit justement l'atome, comme une oscillation entre l'absence et la présence? Invisible à l'œil nu, celui-ci est pourtant le principe même de l'incarnation du monde dans la matière. L'atome explique le personnage, est « [1]a plus petite particule d'existence qui constitue toute forme de matière et la matière dans toutes ses formes » $(A, 12)$. On comprend que cette définition de la pluralité du microscopique constitue le principe même de la poétique scénique privilégiée par la dramaturge dans À tu et à moi. Migneron y reviendra d'ailleurs au tableau 18, alors que les personnages évoquent entre elles le souvenir d'« un casse-tête qu'on essaie d'assembler sans l'image de la boîte pour nous guider » $(A, 45)$. Certes, dans cet exercice théâtral marqué par la vacuité des propos échangés, tous les éléments du récit y sont atomisés, pulvérisés, volatilisés. Mais, du même souffle, cette perte dramatique de la densité permet contre toute attente de libérer « la matière dans toutes ses formes », tout cela qui depuis toujours se blottit dans les jeux inconséquents du langage. En contrepoint du vide apparent, en fait, se blottit l'imaginaire interdit et sans voix qui, de tout temps, cherche à se matérialiser. La matière est là, épiphanique, dans la voix et le geste de l'actrice. Le théâtre restitue à la matière de l'atome toute sa visibilité.

Pour Migneron, le théâtre s'intéresse aux mécanismes mêmes de l'occultation et de l'interdit, sans pour autant autoriser leur renversement. Car le langage sera toujours un tissu de mensonges et la blessure ne cessera de se cicatriser. Au tableau 7 d'À tu et à moi, les deux voix féminines se conjuguent et laissent entrevoir la tristesse déchirante qui les habitent depuis l'enfance. 
FRANÇOIS PARÉ, «Un théâtre d'expression féminine en Ontario français depuis 2008 : étude de trois auteures »

- Puis je commence à pleurer. Je pleure d'abord lentement, doucement, silencieusement. Je pleure les pleurs d'une fille qui n'a jamais voulu laisser entendre ses pleurs. Je m'écoute pleurer en silence. Assise dans le noir. Et je laisse le noir de mes pleurs m'envelopper, me bercer, me percer au même rythme que ma berceuse » $(A, 22)$.

Le premier recel est donc celui-là qui a permis d'occulter la scansion éplorée de la fille, son cri perçant, elle que le langage avait recouverte d'une chape de questions et dont on n'entendait plus la quête du sens.

Dès ce moment, les métaphores de la membrane (peau, pelure, couverture, cavité, sable) pénètrent les dialogues d'À tu et à moi comme autant de fables sur l'interdit. Déjà, la scène des pleurs au tableau 7 s'était terminée sur l'image de l'enfant recroquevillée sous les couvertures, dans « cette chambre froide et frigide et raide et froide et vide » $(A, 22)$. Ces premières évocations avaient alors été suivies par une brève série de questions sur l'identité et surtout par l'épisode récurrent de l'orange transpercée autour duquel se mettent à tournoyer les deux voix de femmes.

Le fruit apparaît d'abord comme un prétexte. Aux tableaux 4 et 15, le personnage identifié par un long tiret offre d'entrée de jeu une orange à son interlocutrice qui, ne sachant pas quoi penser, ne la mangera pas tout de suite. Où pourrait-elle mettre cet objet encombrant dont elle ne saisit pas le sens, pas plus d'ailleurs que le spectateur?

--- Veux-tu y goûter?

- Plus tard.

--- Je la mets où?

- N'importe où.

--- C'est où, n'importe où?

- Là.

Elle n'indique nulle part $(A, 40)$.

L'indifférence des deux femmes trahit pourtant un malaise profond. Car l'orange marque par ailleurs le surgissement de la matière vivante, primordiale, dans le jeu hétéroclite de la voix 
scénique, jusque-là inconséquente et allusive. Le fruit mis à découvert, disséqué et consommé trahit la violence et la coercition explicites du rapport à la matière. Dans ces passages hautement symboliques, le texte de Migneron renvoie au sacrifice initial de la femme :

--- Autre façon de disséquer une orange.

Poser la victime sur une surface plate quelconque : comptoir, planche de bois, couvercle de cercueil.

Pendant qu'une main s'occupe de maintenir l'orange en place, enrouler les doigts de l'autre main autour du manche d'un couteau édenté, pointu et tranchant. Une fois l'orange matée et colmatée, y insérer le couteau. Débuter à la base, la tête, le sommet, le pôle nord des quartiers et traverser l'orange de part en part jusqu'à ce que deux moitiés soient obtenues $(A, 42)$.

Dans ces moments saisissants de la pièce de Sarah Migneron, le spectateur se rend compte de ce qui souffre sous l'interdit. Au tableau 16 d'À tu et à moi le choix stylistique du mode d'emploi s'oppose à la densité métaphorique du rituel au cours duquel la matière de l'orange s'est trouvée fissurée, « colmatée », puis soumise une fois pour toutes au régime de l'ellipse.

Ce qui était «nulle part» est en effet au cœur de l'interdit et du meurtre ritualisé de la naissance qu'il représente. Chez Migneron, la brièveté des dialogues et la juxtaposition de mises en situation hétéroclites contrevient au désir de la voix performative qui souhaiterait plutôt entendre « raconter une histoire pendant longtemps » $(A, 34)$. La dramaturge ne laisse le récit arriver à son dénouement qu'en de très rares occasions. L'œuvre de Migneron montre que la mise en récit de l'identité ne dispose d'aucune légitimité dans cet univers pulvérisé « aux tiroirs pleins de rien et aux souvenirs vides de tout » $(A, 22)$.

\section{Conclusion}

Comme nous l'avons vu, les trois œuvres à l'étude privilégient les formes brèves qui, à divers degrés, semblent plus aptes à rendre compte des interdits, des abjections et des souffrances qui frappent le passé trouble des personnages. L'irruption du tableau ou de la 
FRANÇOIS PARÉ, «Un théâtre d'expression féminine en Ontario français depuis 2008 : étude de trois auteures »

scène suscite de l'inattendu, du hasard. Une révélation, peut-être. Puisque la mise en scène de la mémoire est toujours différée sur le plan symbolique, la trame narrative semble désorientée, marquée par l'inachèvement. Le passé se présente comme une matière trouée. Il en résulte chez Anne-Marie White, Lisa L'Heureux et surtout Sarah Migneron une véritable crise du dialogue: paroles sans réponse, parallélismes, coqs-à-l'âne et déclarations inachevées. L'analepse sur laquelle se fonde la quête de rédemption reste le plus souvent une plaie ouverte. Vulnérables, les personnages apparaissent et disparaissent ainsi au gré de scènes succinctes qui permettent le dévoilement graduel de la vérité sans pour autant combler les interstices. La parole performative constitue la seule armature des personnages qui cherchent, à chaque instant, à mettre au grand jour les violentes tensions qui les obsèdent. Cependant, chacun de ces êtres stigmatisés par la violence « évite de se mesurer directement à l'autre, privilégiant, face au public, le récit des manifestations intérieures qui l'habitent » (Hébert et Perello-Contos, 2004, p. 34). Ce jaillissement du récit, parfois entravé par la souffrance muette des personnages, confirme ainsi l'engagement du théâtre franco-ontarien au féminin envers une plus grande «individualisation des pratiques théâtrales », selon l'expression proposée par Jane Moss. (2010, p. 83)

Les œuvres que nous avons parcourues ne rompent pas tout à fait avec la trame identitaire du théâtre franco-ontarien de la fin du $\mathrm{XX}^{\mathrm{e}}$ siècle. Toutefois, le personnage de la femme « abîmée » y occupe maintenant une très large part. Délesté du poids de l'identité collective, le sujet féminin s'est emparé de la voix performative. Telle aura été la stigmatisation de l'enfance qu'elle aura permis le dévoilement des mécanismes mêmes de l'occultation. L'objectif de ce théâtre est donc celui que fixait, dans Lumières du corps, Valère Novarina, comme une caractéristique fondamentale de l'expérience scénique : 
le théâtre est un lieu de perdition où nous refaisons l'expérience effrayante du chaos, où nous sommes décomposés, le lieu de la défaite humaine : le lieu où mous venons ensemble nous déconstruire : c'est un lieu commun où nous nous rassemblons pour qu'éclatent tous les lieux communs. (2006, p. 67)

Dans les trois œuvres étudiées, ce lieu commun est aussi celui de la stigmatisation de l'enfance et de la structure elliptique et fragmentaire de l'espace scénique.

\section{Bibliographie}

DEGLISE, Fabien, (11 octobre 2013), « Théâtre - Descente dans la mésestime du soi », Le Devoir, disponible en ligne : https://www.ledevoir.com/culture/theatre/389764/descentedans-la-mesestime-du-soi.

FRIEND, David, « Tom Cochrane reflects on success of "Life is a Highway" », The Canadian Press et The Toronto Star, 5 janvier 2017, en ligne:

https://www.thestar.com/entertainment/music/2017/01/05/tom-cochrane-reflects-onsuccess-of-life-is-a-highway.html.

GoFFI, Emmanuel, « Pour l'hiver : acte de naissance du talent littéraire de Lisa L'Heureux », (blogue), Apt613, 2 mars 2017, en ligne : URL https://apt613.ca/pour-lhiveracte-de-naissance-du-talent-litteraire-de-lisa-lheureux/.

GUAY, Hervé, «De la polyphonie hétéromorphe à une esthétique de la divergence », L'Annuaire théâtral, $\mathrm{n}^{\mathrm{o}}$ 47, printemps 2010, p. 15-36.

HÉBERT, Chantal, et Irène PERELLI-CONTOS (dir.), La narrativité contemporaine au Québec. Le théâtre et ses nouvelles dynamiques narratives, Québec, Presses de l'Université Laval, 2004.

HotTe, Lucie, et François OUELLET (dir.), La littérature franco-ontarienne depuis 1996. Nouveaux enjeux esthétiques, Sudbury, Prise de parole, 2016.

LECLERC, Yves, «La folie du quotidien », Le Journal de Québec, 8 octobre 2016, en ligne : https://www.journaldequebec.com/2016/10/08/la-folie-du-quotidien.

LÉVESQUE, François, « Cri du cœur du Conseil québécois du théâtre », Le Devoir, 5 avril 2013, en ligne : https://www.ledevoir.com/culture/theatre/374982/cri-du-coeur-du-conseilquebecois-du-theatre.

L'Heureux, Lisa, Pour l'hiver, Sudbury, Prise de parole, 2016.

Migneron, Sarah, À tu et à moi, Sudbury, Prise de parole, 2015. 
FRANÇOIS PARÉ, «Un théâtre d'expression féminine en Ontario français depuis 2008 : étude de trois auteures »

Moss, Jane, « Le théâtre francophone en Ontario », dans Lucie Hotte et Johanne Melançon (dir.), Introduction à la littérature franco-ontarienne, Sudbury, Prise de parole, 2010, p. 71106.

NoLETTE, Nicole, Jouer la traduction. Théâtre et hétérolinguisme au Canada francophone, Ottawa, Presses de l'Université d'Ottawa, 2015.

NovarinA, Valère, Lumières du corps, Paris, P.O.L., 2006.

NutTing, Stéphanie, « Joël Beddows, agent double », Francophonies d'Amérique, n 33, printemps 2012, p. 13-35.

PASQUIER, Dominique, «Sociabilités et sorties au théâtre », Culture études, vol. 1, no 1, 2013, p. 1-12, disponible en ligne : https://www.cairn.info/revue-culture-etudes-2013-1page-1.htm.

REISING, Russell, Loose Ends : Closure and Crisis in the American Social Text, Durham, Duke University Press, 1996.

ROBERT, Lucie, « Théâtre et féminisme au Québec », Québec français, $\mathrm{n}^{\circ}$ 137, printemps 2005, p. 43-46.

RUPRECHT, Alvina, « À tu et à moi : une présence post-moderne qui remonte aux origines de la danse moderne », Le Cercle des critiques de la Capitale (blogue), 23 septembre 2013, en ligne : ,http://capitalcriticscircle.com/tu-et-moi/,

RYNGAERTS, Jean-Pierre, « Le personnage théâtral contemporain : symptôme d'un nouvel "ordre dramaturgique" », L'Annuaire théâtral, no 43-44, printemps-automne 2008, p. 103112.

SEPINUCK, Teya, Theater of Witness : Finding the Medicine in Stories of Suffering, Transformation and Peace, Londres, Jessica Kingsley Publishers, 2013.

ThiBAult, Laurence V., « Anne-Marie White. Le portrait blanc-bleu », Liaison, n 140, 2008, p. 12-13.

White, Anne-Marie, Déluge, Sudbury, Prise de parole, 2013.

\section{Résumé}

Dans cette étude de trois dramaturges de la relève féminine en Ontario français, Anne-Marie White, Lisa L'Heureux et Sarah Migneron, nous sommes à même de constater l'émergence d'éléments formels qui repoussent l'horizon du théâtre franco-ontarien, tel qu'il s'était dessiné depuis les premières pièces d'André Paiement au début des années 1970 jusqu'aux œuvres marquantes de Jean Marc Dalpé, Robert Marinier et Michel Ouellette. Les œuvres de White, L'Heureux et Migneron sont dénuées de toute référence à l'Ontario français. Malgré 
la persistance du drame familial, on y assiste plutôt à une reformulation du spectacle scénique, désormais façonné par la perte de cohérence de l'aventure introspective de chacun des personnages dans sa solitude existentielle. La structure actantielle et la trame narrative supposent dès lors la mise en présence d'individualités orphelines, certes victimes d'ostracisme et de violence, mais sans autre distinction que le régime pronominal lui-même auquel s'assujettit une mémoire marquée par le traumatisme.

\begin{abstract}
This study of three new female Franco-Ontarian playwrights, Anne-Marie White, Lisa L'Heureux and Sarah Migneron, seeks to trace the emergence of notable stylistic elements that push back the horizon of the French-language stage in Ontario, as it moves away from André Paiement's first plays in the early 1970s and the remarkable works of Jean Marc Dalpé, Robert Marinier and Michel Ouellette. The works of White, L'Heureux and Migneron are devoid of any references to Franco-Ontarian identity. Despite the persistence of the family drama, we are witnessing a rephrasing of the theatrical space itself and a loss of coherence in the introspective self-narrative of the individual characters' existential solitude. The actantial structure reveals the presence of orphaned figures, who, while the victims of ostracism and violence, are stripped of any other distinction than the pronominal regime itself and the traumatic memory it represents.
\end{abstract}

\title{
Notice biobibliographique
}

François Paré est Distinguished Professor emeritus au département d'études françaises de l'Université de Waterloo (Ontario) et membre de la Société Royale du Canada. En 1993, son livre Les littératures de l'exiguité lui a valu le Prix du Gouverneur Général du Canada. Il est aussi l'auteur de Théories de la fragilité (Le Nordir, 1994) et de La distance habitée (Le Nordir, 2003). Il a aussi fait paraître Le fantasme d'Escanaba (Nota bene 2008) et, avec François Ouellet, un essai sur le romancier québécois Louis Hamelin (Nota bene 2008). Il a récemment dirigé en compagnie de Lucie Hotte Les littératures francophones minoritaires au Canada à l'aune du temps (Presses de l'Université d'Ottawa, 2016) et fait paraître depuis 2015 un certain nombre d'études sur les premiers écrits français de la région des Grands Lacs au Canada (1630-1760). 